\title{
A CHARACTERIZATION OF SINGULAR ENDOMORPHISMS OF A BARRELLED PTÁK SPACE
}

\author{
DAMIR FRANEKIĆ \\ 1304 Spring Street \\ Bethlehem, PA 18018 \\ U.S.A. \\ (Received October 29, 1980 and in revised form July 2, 1981)
}

\begin{abstract}
The concept of topological divisor of zero has been extended to endomorphisms of a locally convex topological vector space (LCTVS). A characterization of singular endomorphisms, similar to that of Yood [1], is obtained for endomorphisms of a barrelled Pták (fully complete) space and it is shown that each such endomorphism is a topological divisor of zero. Furthermore, properties of the adjoint of an endomorphism are characterized in terms of topological divisors of zero, and the effect of change of operator topology on such a characterization is given. KEY WORDS AND PHRASES. Singular endomorphisms, topological divisors of zero, locally convex barrelled Pták spaces.

AMS (MOS) SUBJECT CLASSIFICATION (1980). Primary 46H05, $46 \mathrm{H} 20$.
\end{abstract}

\section{INTRODUCT ION.}

The reader should be familiar with barrelled spaces and have available the four references listed. The following notation and definitions will be used. $(X, T)$ is an LCTVS over a field $K$ of complex numbers, $X^{\prime}$ is its topological dual, and $C(X, T)$ the algebra of all the $T$-continuous endomorphisms of $X . w=\sigma\left(X, X^{\prime}\right)$ is the weak topology on $X$ by $X^{\prime}, w^{*}=\sigma\left(X^{\prime}, X\right)$, and $\beta^{\prime}$ is the topology on $X^{\prime}$ of uniform convergence on all the w-bounded subsets of $X--$ the strong topology.

$C(X, T) \subseteq C(X, w)$ can be made into a topological space in a number of ways. If $A$ is a family of w-bounded (hence bounded) subsets of $X$ and $N=N(T)$ is a $T$-neighborhood base at zero, then the sets 


$$
\mathrm{N}(\mathrm{A}, \mathrm{V})=\{\mathrm{T} \varepsilon \mathrm{C}(X, T): \mathrm{TA} \subseteq \mathrm{V}\},
$$

where $A$ and $V$ run through $A$ and $N$ respectively, form a neighborhood base at zero for a locally convex vector topology $\bar{T}(A, T)$ on $C(X, T)$, the operator topology of uniform convergence on members of $A$ relative to $T$. The space $C\left(X^{\prime}, w^{*}\right) \subseteq C\left(X^{\prime}, \beta^{\prime}\right)$ can be topologized in a similar manner. For each $T \varepsilon C(X, w)$ its adjoint is a w*continuous endomorphism on $X^{\prime}$, i.e. $T \varepsilon C\left(X^{\prime}, w^{*}\right) \subseteq C\left(X^{\prime}, \beta^{\prime}\right)$. Let $A$ be a family of bounded subsets of $X$ which contains the family $F$ of all the finite subsets of $X$. The topology $T_{A}$ on $X^{\prime}$ of uniform convergence on the members of $A$ is then stronger than $w^{*}$, in fact it is between $w^{*}$ and $B^{\prime}$. If for each $T \varepsilon C(X, w)$, TA $\subseteq A$ then $C\left(X^{\prime}, w^{*}\right) \subseteq C\left(X^{\prime}, T_{A}\right) \subseteq C\left(X^{\prime}, B^{\prime}\right)$. If $A=F, \bar{T}(F, T)$ is the operator topology of pointwise convergence relative to $T$ while in the case of $A=B--$ the class of all wbounded subsets of $X, \vec{T}(B, T)$ is the strong operator topology relative to $T$. Finally if $A \subseteq X, A^{O} \subseteq X^{\prime}$ is its absolute polar, similarly $B_{O}$ is the absolute polar, in $X$, of $\mathrm{B} \subseteq X^{\prime}$.

Given $T \varepsilon(C(X, T), \bar{T}(A, T))$ and $(D, \leq)$ a directed set, the following definition extends the concept of topological divisors of zero (tdz) to $C(X, T)$.

DEFINITION 1. T is a left (right) topological divisor of zero, 1 tdz(rtdz), if there is a net $\left\{s_{\delta}: \delta \varepsilon D\right\} \subseteq \mathcal{C}(X, T)$ which doesn't converge to zero, written $s_{\delta} \rightarrow 0$, yet the net $\mathrm{TS}_{\delta}\left(\mathrm{S}_{\delta} \mathrm{T}\right)$ does converge to zero, written $\mathrm{TS}_{\delta} \rightarrow 0\left(\mathrm{~S}_{\delta} \mathrm{T} \rightarrow 0\right)$, in $\bar{T}(\mathrm{~A}, T)$.

REMARK. This means, there are $A^{\prime} \varepsilon A$ and $V^{\prime} \varepsilon N$ such that $S_{\delta} A^{\prime} \ddagger V^{\prime}$ frequently, yet for all $A \in A$ and $V \in N$, TS $\delta^{A} \subseteq$ ( $\left.S_{\delta} T A \subseteq V\right)$ eventually.

Following Yood [1] we use $z^{l}\left(z^{r}\right)$ to denote the sets of all left (right) tdz and $H^{l}\left(H^{r}\right)$ their respective complements in $C(X, T)$. Furthermore, $G^{l}\left(G^{r}\right)$ will mean the sets of all left (right) regular elements of $C(X, T)$ and $S^{l}\left(S^{r}\right)$ their complements. Finally, $N^{l}\left(N^{r}\right)$ are the sets of all left (right) divisors of zero -- $1 \mathrm{dz}$ $(\operatorname{rd} z)$.

\section{BASIC RESULTS.}

It can easily be seen that all the basic properties of tdz remain valid as in the case of a Banach space. Some of them are listed in the following lemma.

LEMMA 1. The following inclusions are valid in $C(X, T)$ : 

a) $N^{l} \subseteq Z^{l}, N^{r} \subseteq Z^{r}$.
b) $z^{l} \leq s^{l}, z^{r} \leq s^{r}$.
c) $G^{l} \subseteq H^{r}, G^{r} \subseteq H^{l}$.
d) $G^{l} \cap S^{\mathrm{r}} \subseteq N^{\mathrm{r}}, G^{\mathrm{r}} \cap S^{l} \subseteq N^{l}$.
e) $G=G^{l} \cap G^{r} \subseteq G^{l} \cap H^{r} \subseteq G^{r} \cap H^{l}$.

A slight modification in Yood's proof of Theorems 3.1 and 3.2 ([1], p. 493) yields the following result.

PROPOSITION 1.
a) $N^{\ell}=\{\mathrm{T}: \quad \mathrm{T}$ is not injective $\}$.
b) $N^{r}=\{\mathrm{T}: \overline{\mathrm{TX}} \neq \mathrm{X}\}$.

We shall refer to $T \varepsilon C(X, T)$ as to a topological isomorphism if $T$ is injective and $T$-relatively open as a map from $X$ onto $T X$.

For $y \varepsilon X$ and $x^{\prime} \varepsilon X^{\prime}$ we define $y \bigotimes x^{\prime} \varepsilon C(X, T)$ by $y \bigotimes x^{\prime}(x)=x^{\prime}(x) y$.

The next theorem characterizes topological isomorphism in terms of $t d z$.

THEOREM 1. TEC $(X, T)$ is a T-topological isomorphism iff $\left.\mathrm{TEH}^{\ell} \subseteq(C(X, T)), \bar{T}(A, T)\right)$.

PROOF. A T-topological isomorphism can not be an $1 \mathrm{tdz}$. For if it were with $\left\{\mathrm{S}_{\delta}\right\}, \mathrm{A}^{\prime}$ and $\mathrm{V}^{\prime}$ as in Remark after Definition $1, \mathrm{~S}_{\delta} \mathrm{A}^{\prime} \ddagger \mathrm{V}^{\prime}$ frequently yet $\mathrm{TS}_{\delta} \mathrm{A} \subseteq \mathrm{V}$ eventually for all $A \varepsilon A$ and $V \varepsilon N$, particularly for $U=T^{\prime} \varepsilon N$ because $T$ is open. This would, however, imply that $\mathrm{TS}_{\delta} \mathrm{A}^{\prime} \subseteq \mathrm{U}=\mathrm{TV}^{\prime}$ eventually which is impossible. If $\mathrm{T}$ is not a T-topological isomorphism, $T$ is either not injective, or $T$ is injective but $\mathrm{T}^{-1}$ is not $T$-relatively open. $\mathrm{T}$ being not injective implies, accordingly to Lemma 1a, $T \in N^{l} \subseteq Z^{l}$. On the other hand, $\mathrm{T}^{-1}$ being not $T$-relatively open implies the existance of a net $\left\{y_{\delta}\right\} \subseteq T X$ and the net $\left\{x_{\delta}=T^{-1} y_{\delta}\right\}$ with the property that $y_{\delta} \rightarrow 0$ and $x_{\delta} \nrightarrow 0$ in $T$. Let $0 \neq x^{\prime} \varepsilon X^{\prime}$ and construct the endomorphisms $s_{\delta}=x_{\delta}(y.) x^{\prime}$. Then the net $\left\{s_{\delta}\right\} \subseteq C(X, T)$ is such that $s_{\delta} \rightarrow 0$ yet $\mathrm{TS}_{\delta} \rightarrow 0$ in $\bar{T}(A, T)$. To see this, take $A \varepsilon A$ and $V \varepsilon N$, then $T_{\delta} A=x^{\prime}(A) y_{\delta}$. T-boundedness of A and T-continuity of $x^{\prime}$ imply that $M=\sup \left\{\left|\left\langle a, x^{\prime}\right\rangle\right|: a \varepsilon A\right\}$ exists. Then $y_{\delta} \neq 0$ implies that $y_{\delta} \varepsilon M^{-1} V$ eventually, hence $\mathrm{TS}_{\delta} \mathrm{A} \subseteq \mathrm{V}$ eventually.

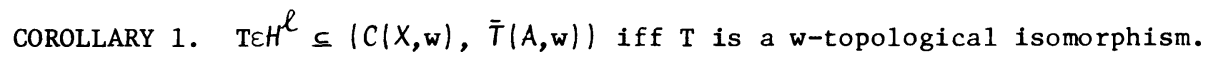
THEOREM 2. $T \varepsilon C(X, w)$ is surjective iff $\operatorname{TEH}^{\mathrm{r}} \subseteq(C(X, w), \bar{T}(F, T))$. 
PROOF. Let $T$ be surjective. If $T E Z^{r} \subseteq(C(X, w), T(F, T))$, then with $S_{\delta}$, $A^{\prime}=\Gamma_{E} F$ and $V^{\prime} \in N$ as in Remark, we have $S_{\delta} F \notin V^{\prime}$ frequently. Since $S_{\delta} T F \subseteq V$ eventually for all $F E F$ and $V E N, S_{\delta} T F_{1}=S_{\hat{\delta}} F \subseteq V^{\prime}$ eventually for $F_{1}=T^{-1} F$, which contradicts the choice of the net $S_{\delta}$.

Conversely, assume $\mathrm{T}$ is not surjective. In the case that its range is not dense in $X$, according to Lemma $1 \mathrm{~b}, T \varepsilon N^{\mathrm{r}} \subseteq Z^{\mathrm{r}}$. If however the range of $\mathrm{T}$ is dense in $X$, its adjoint $T^{\prime}$ is injective but not $w^{*}$-relatively open, hence there is a net $\left\{y_{\delta}^{\prime}\right\} \subseteq T^{\prime} X^{\prime}$ and the net $\left\{x^{\prime}=\left(T^{\prime}\right)^{-1} y_{\delta}^{\prime}\right\}$ such that $y_{\delta}^{\prime} \rightarrow 0$ in $w^{*}$-topology yet $x_{\delta}^{\prime} f \rightarrow 0$. Let $0 \neq x_{0} \varepsilon X$, and construct the endomorphisms $s_{\delta}=x_{0} \otimes x_{\delta}^{\prime} \varepsilon \mathcal{C}(X, T)$. The net $\left\{\mathrm{s}_{\delta}\right\}$ has the property that $\mathrm{s}_{\delta} \rightarrow 0$ in $\bar{T}(F, T)$ yet $\mathrm{s}_{\delta} \mathrm{T} \rightarrow 0$ in the same topology. The map $(\lambda, x) \rightarrow \lambda x$ is separately continuous. This proves that $T \in Z^{r}$.

The next four lemmas will be used to sharpen the results obtained so far.

LEMMA 2. A relatively open, continuous endomorphism of a barrelled space must have a barrelled range.

PROOF. If $T X$ is not barrelled there is a net $\left\{y_{\delta}\right\} \subseteq T X$ which tends to zero and doesn' $t$ belong to a barrel $B$ in $T X$. Then the net $\left\{x_{\delta}=T^{-1} y_{\delta}\right\}$ can not tend to zero because it is not eventually in the neighborhood $U=T^{-1}$.

LEMMA 3. Any relatively open endomorphism $T$ of a complete space must have a closed range.

PROOF. Let $\left\{\mathrm{y}_{\delta}\right\}$ be a net in the range of $\mathrm{T}$ and let $\mathrm{y}_{\delta} \rightarrow \mathrm{y}$. Since $\left\{\mathrm{y}_{\delta}\right\}$ is a Cauchy net and $T$ is relatively open the net $\left\{x_{\delta}=T^{-1} y_{\delta}\right\}$ is also a Cauchy net hence converges to some $\mathrm{x} \varepsilon X$. Then $\mathrm{Tx}_{\delta}=\mathrm{y}_{\delta} \rightarrow \mathrm{Tx}=\mathrm{y}$, hence the range of $\mathrm{T}$ is closed.

LEMMA 4. Let $T$ be an endomorphism of a barrelled Pták space $(X, T)$. If its adjoint $T^{\prime}$ is $\beta^{\prime}$-topological isomorphism, then $T$ is surjective and open.

PROOF. Let $M$ be a balanced, convex, $w^{*-c l o s e d ~ a n d ~ T-e q u i c o n t i n u o u s ~ s u b s e t ~ o f ~}$ $X^{\prime}$. It is then both $w^{*}$-compact and $\beta^{\prime}$-bounded. Since $T^{\prime}$ is $\beta^{\prime}$-open, $\left(T^{\prime}\right)^{-1} M=B$ is $\beta^{\prime}$-bounded. $B$ is also $w^{*}$-closed because $T^{\prime}$ is $w^{*}$-continuous. Since $(X, T)$ is barrelled, $B$ is $w^{*}$-compact and this in conjunction with $w^{*}$-continuity of $\mathrm{T}^{\prime}$ implies that $T^{\prime} B=T^{\prime} X^{\prime} \cap M$ is $w^{*}$-compact, hence $w^{*}$-closed. Since $(X, T)$ is also a Pták space it implies that $\mathrm{T}$ is relatively open. Finally, completeness of a Pták space 
and injectiveity of $\mathrm{T}^{\prime}$ imply that $\mathrm{T}$ is both closed (Lemma 3 ) and dense in $X$, hence $\mathrm{T}$ is surjective.

LEMMA 5. If $T \varepsilon C(X, T)$ is surjective and $T$-open, then $T \varepsilon H^{r} \subseteq(C(X, T), \bar{T}(A, T))$.

PROOF. If $T \varepsilon Z^{r}$, then with $S_{\delta}, A^{\prime} \varepsilon A$ and $V^{\prime} \varepsilon N$ as in Remark, $S_{\delta} A^{\prime} \ddagger V^{\prime}$ frequent1y. $\mathrm{T}^{-1} \mathrm{~A}^{\prime}=\mathrm{A}_{1}$ is bounded, because $\mathrm{T}$ is open. Since $\mathrm{S}_{\delta} \mathrm{T} \rightarrow 0$ in $\bar{T}(A, T), \mathrm{S}_{\delta} \mathrm{TA}_{1} \subseteq \mathrm{V}^{\prime}$ eventually and this is impossible because $\mathrm{S}_{\delta} \mathrm{TA}_{1}=\mathrm{S}_{\delta} \mathrm{A}^{\prime}$, hence $\mathrm{T} \varepsilon H^{\mathrm{r}}$.

THEOREM 3. If $(X, T)$ is a barrelled Pták space, then $T \varepsilon H^{l} \subseteq(C(X, T), \bar{T}(A, T))$

iff $\mathrm{T}$ is injective and $\mathrm{T} X$ is barrelled.

PROOF. A T-continuous injection from a Pták space into a barrelled space is a T-topological isomorphism, hence according to Theorem 1, T\& $H^{\ell}$. The converse follows from Lemma 2 and Theorem 1 .

THEOREM 4. If $(X, T)$ is a barrelled Pták space, then $\operatorname{TEH}^{\mathrm{r}} \subseteq(\mathcal{C}(X, T), \bar{T}(A, T))$ iff $\mathrm{T}$ is surjective.

PROOF. If $\mathrm{T}$ is surjective then, according to Proposition 2 [3, p. 299], $\mathrm{T}$ is T-open hence by Lemma 5 it cannot be a rtdz.

Assume now that $\mathrm{T}$ is not surjective. If the range of $\mathrm{T}$ is not dense, then according to Lemma $1 . a, T \in N^{r} \subset Z^{r}$. Suppose that the range of $T$ is dense in $X$. $T$ can not be T-open, because it would have to have a closed range (Lemma 3). According to Lemma 4, its adjoint $\mathrm{T}^{\prime}$ (which is injective) can not be $\beta^{\prime}$-relatively open, hence there is a net $\left\{y_{\delta}^{\prime}\right\} \subseteq T^{\prime} X^{\prime}, y_{\delta}^{\prime} \rightarrow 0$ and the net $\left\{x_{\delta}^{\prime}=\left(T^{\prime}\right)^{-1} y_{j}^{\prime}\right\} \rightarrow 0$ in $B^{\prime}$. The conclusion then follows just as in the last part of Theorem 2 .

Since Fréchet space is both barrelled and Pták space, Theorems 3 and 4 are valid for them.

COROLLARY 2. If $(X, T)$ is a Fréchet space then

a) $\mathrm{T}$ is injective and range closed iff $\mathrm{T} \in H^{\ell} \subseteq(\mathrm{C}(X, T), \bar{T}(A, T))$.

b) $\mathrm{T}$ is surjective iff $T \varepsilon H^{\mathrm{r}} \subseteq(C(X, T), \tilde{T}(A, T))$.

PROOF. Part a) follows from the fact that a closed subspace of an Fréchet space is a Fréchet space, hence barrelled and from Theorem 3. Part b) follows from Theorem 4 and the foregoing remark.

Properties of a linear operator on an LCS are very intimately related to those 
of its adjoint operator. For example: "T is w-open iff $T$ ' is $w^{*}$-range closed".

The next theorem relates properties of $T$ with those of $T^{\prime}$ in terms of $t d z$. In this regard it is important to note that an operator topology $\bar{T}(A, T)$ remains unaltered if either $A$ is replaced with its balanced convex and closed envelope and/or $N$ is replaced with a fundamental system of balanced, convex and closed neighborhoods. In the sequel it is assumed that each $A \varepsilon A$ and $V \varepsilon N$ is balanced convex and closed.

THEOREM 5. Let $A$ be a family of bounded subsets of $X$ and $T E C(X, w)$ such that $\mathrm{TA} \subseteq \mathrm{A}$. Then the following are equivalent:

a) $\mathrm{T} \varepsilon Z^{\ell}\left(Z^{\mathrm{r}}\right) \subseteq(C(X, w), \bar{T}(A, T))$.

b) $T^{\prime} \varepsilon Z^{r}\left(Z^{\ell}\right) \subseteq\left(C\left(X^{\prime}, w^{*}\right), \bar{T}\left(E, T_{A}\right)\right)$, where $E$ is the set of all $T$ equicontinuous subsets of $X^{\prime}$.

PROOF. The condition $T A \subseteq A$ makes $T^{\prime} T_{A}$-continuous. This together with the $T_{A}$-boundedness of each EEE implies that $\bar{T}\left(E, T_{A}\right)$ is a vector topology. The statement then follows from the following facts:

- $T A \subseteq B$ iff $T^{\prime} B^{O} \subseteq A^{\circ}$, if $A, B$ are convex, balanced and closed;

$-\mathrm{S}_{\delta} \rightarrow 0$ in $\bar{T}(A, T)$ iff $S_{\delta}^{\prime} \rightarrow 0$ in $\bar{T}\left(A, T_{A}\right)$;

$-\left(S_{\delta} T\right)^{\prime}=T^{\prime} S_{\delta}^{\prime}$.

In what will follow $B^{\prime}$ and $F^{\prime}$ will denote the sets of all $w^{*}$-bounded and finite subsets of $X^{\prime}$ respectively.

There is a number of families of banded subsets (equivalently w-bounded) of $X$ which satisfy the condition of Theorem 5 for all $T \varepsilon C(X, w)$. Consider the most extreme ones: F--the family of all finite--and $B--a 11$ bounded--subsets of $X$. Note that $F$ generates the $w^{*}$-topology and $B$ the $\beta^{\prime}$-topology in $X^{\prime}$. In $C(X, w)$, since $F \subseteq B, \bar{T}(F, w)<\bar{T}(B, w)$. The effect of change of operator topology on the results of the preceding theorem are given in the following corollary.

COROLLARY 3. If $\operatorname{TEC}(\mathrm{X}, \mathrm{w})$, the following statements are valid:

a) $\operatorname{T\varepsilon } Z^{\ell}\left(Z^{r}\right) \subseteq(C(X, w), \bar{T}(F, w))$ iff $T^{\ell} Z^{r}\left(Z^{\ell}\right) \subseteq\left(C\left(X^{\prime}, w^{*}\right), \bar{T}\left(F^{\prime}, w^{*}\right)\right)$.

b) $\quad T \in Z^{\ell}\left(Z^{r}\right) \subseteq(C(X, w), \bar{T}(B, w))$ iff $T^{\ell} Z^{r}\left(Z^{\ell}\right) \subseteq\left(C\left(X, w^{*}\right), \bar{T}\left(F^{\prime}, \beta^{\prime}\right)\right)$.

c) $\quad T \varepsilon Z^{\ell}\left(Z^{r}\right) \subseteq(C(X, w), \bar{T}(B, T))$ iff $T \varepsilon Z^{r}\left(Z^{\ell}\right) \subseteq\left(C\left(X^{\prime}, w^{*}\right), T\left(E, B^{\prime}\right)\right)$. 
The next theorem characterizes $w$-isomorphisms and their adjoint in terms of $t d z$. THEOREM 6. If $T \varepsilon C(X, w)$, the following are equivalent:
a) $\mathrm{T}$ is a w-topological isomorphism.
b) $T^{\prime}$ is surjective.
c) $\mathrm{T} \varepsilon H^{l} \subseteq(C(X, w), \bar{T}(B, w))$.
d) $T^{\prime} \varepsilon H^{r} \subseteq\left(C\left(X^{\prime}, w *\right), \bar{T}\left(F^{\prime}, \beta^{\prime}\right)\right)$

PROOF. The equivalence of a) and b) is a standard result and could be found for example in [2, Proposition 8.6.3, p. 517]. The equivalence of a) and c) follows from Corollary 1 while that one of c) and d) from Corollary $3 b$.

The preceeding results can be strengthened in the case of Fréchet space due to the fact that: " $\mathrm{T}$ is an isomorphism iff it is a w-isomorphism" [2, Theorem 8.6.13, p. 521].

COROLLARY 4. If $(X, T)$ is a Fréchet space, the following are equivalent:
a) $\mathrm{T} \varepsilon Z^{\ell} \subset(C(X, T), \bar{T}(B, w))$.
b) $T \varepsilon Z^{\ell} \subset(C(X, T), \bar{T}(B, T))$.
c) $T^{\prime} \varepsilon Z^{r} \subset\left(C\left(X^{\prime}, w^{*}\right), \bar{T}\left(F^{\prime}, \beta^{\prime}\right)\right)$.
d) $T^{\prime} \varepsilon Z^{r} \subset\left(C\left(X^{\prime}, w^{*}\right), \bar{T}\left(B^{\prime}, B^{\prime}\right)\right)$.

PROOF. The equivalence of a) and b) is by Theorem 6 , the foregoing remark and Theorem 1. c) is equivalent to d) because in the dual of a barrelled space $E=B^{\prime}=F^{\prime}$. Finally, b) is equivalent to d) by Corollary $3 c$.

The next theorem generalizes the following result of Rickart [4, p. 297]:

"A singular endomorphism of a Banach space is a topological divisor of zero."

THEOREM 7. Every singular endomorphism of a barrelled Pták space is a topological divisor of zero.

PROOF. T is singular iff $\mathrm{T}$ is elther not injective in which case $\mathrm{TEN} \in \mathrm{N}^{l}$, or $\mathrm{T}$ is not surjective. In the latter case, if the range of $\mathrm{T}$ is not dense, $T \in N^{r} \subseteq Z^{r}$. If however, the range of $T$ is dense then according to Theorem $4, T \varepsilon Z^{r}$. 3. CONCLUSION.

Preliminary results, obtained in this paper, indicate that the concept of $t \mathrm{dz}$ can successfully be used to classify endomorphisms of LCS which are of a more gen- 
eral nature than is Banach space.

A decomposition of $C(X, T)$ into nine disjoint subsets such as in Theorem 3.14, [1], has not been attempted. The conjecture is that it is possible.

A difficult question seems to be the one in regard to a topological characterization of the set of regular endomorphisms and others. An answer to it seems to be directly related to the question:

Under which conditions is $C(X, T)$ a topological algebra with a continuous inverse?

ACKNOWLEDGEMENT. This is an extended version of a portion of the author's dissertation written under Professor A. Wilansky at Lehigh University.

\section{REFERENCES}

1. YOOD, B. Transformations between Banach space in the uniform topology, Annals of Mathematics, Vol. 50, No. 2, April 1949.

2. EDWARDS, R.E. Functional Analysis. Holt, Rinehart and Winston, 1965.

3. HORVATH, J. Topological Vector Spaces and Distributions, Vo1. I. AddisonWesley Publishing Company.

4. RICKART, C.E. General Theory of Banach Algebras. D. Van-Nostrand Co. 


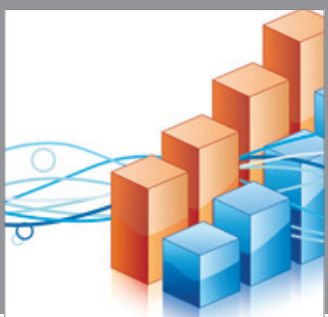

Advances in

Operations Research

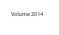

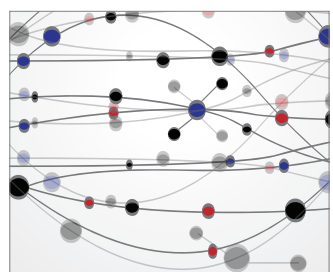

\section{The Scientific} World Journal
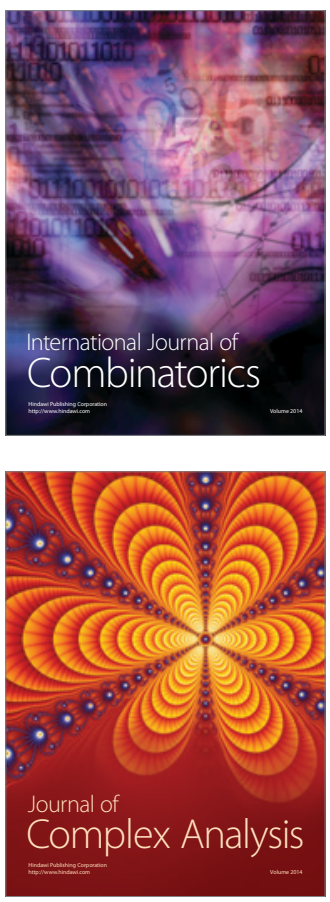

International Journal of

Mathematics and

Mathematical

Sciences
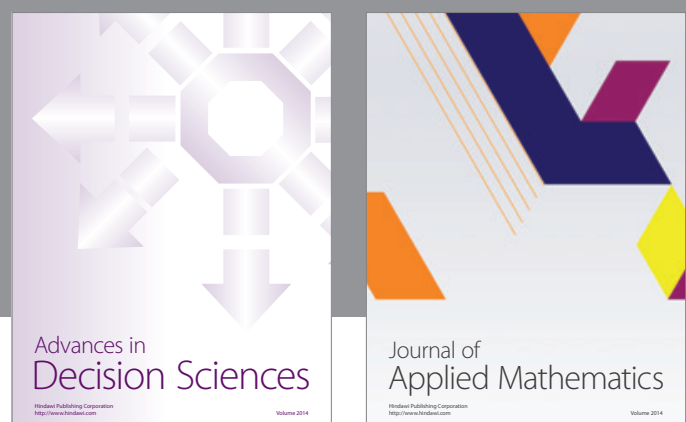

Journal of

Applied Mathematics
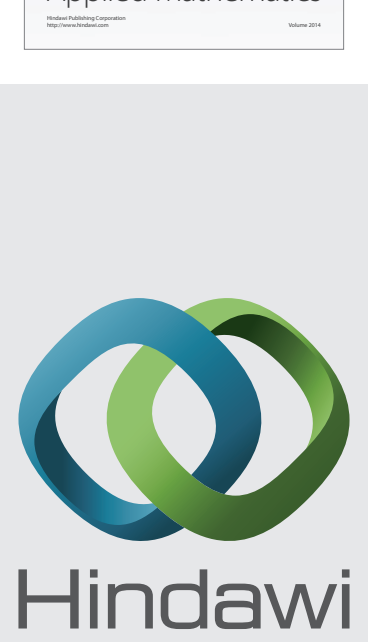

Submit your manuscripts at http://www.hindawi.com
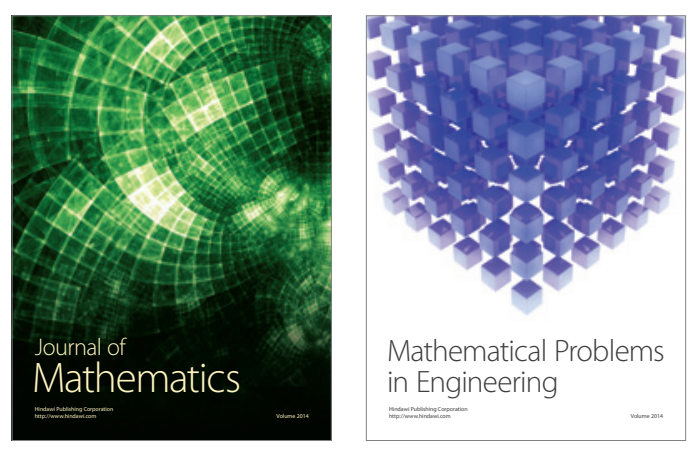

Mathematical Problems in Engineering
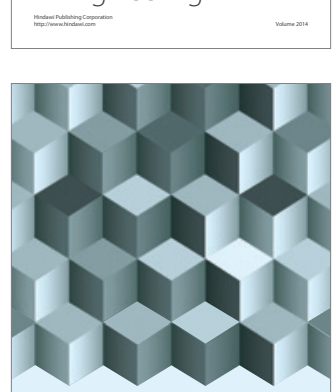

Journal of

Function Spaces
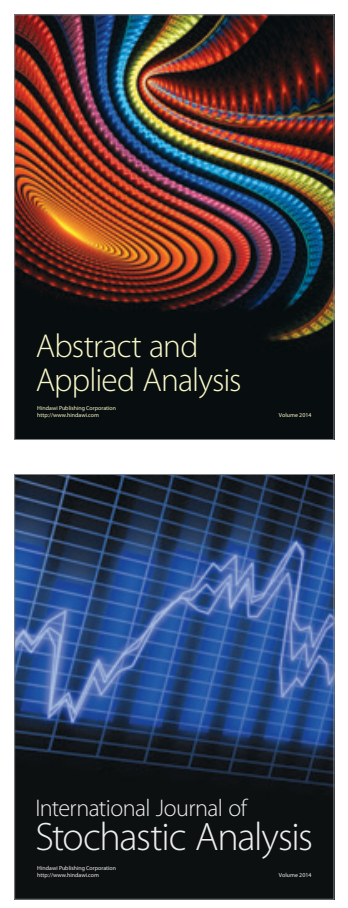

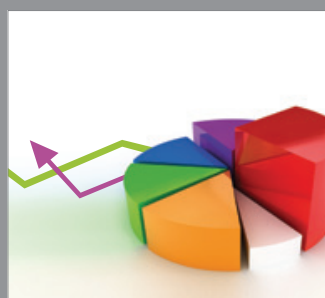

ournal of

Probability and Statistics

Promensencen
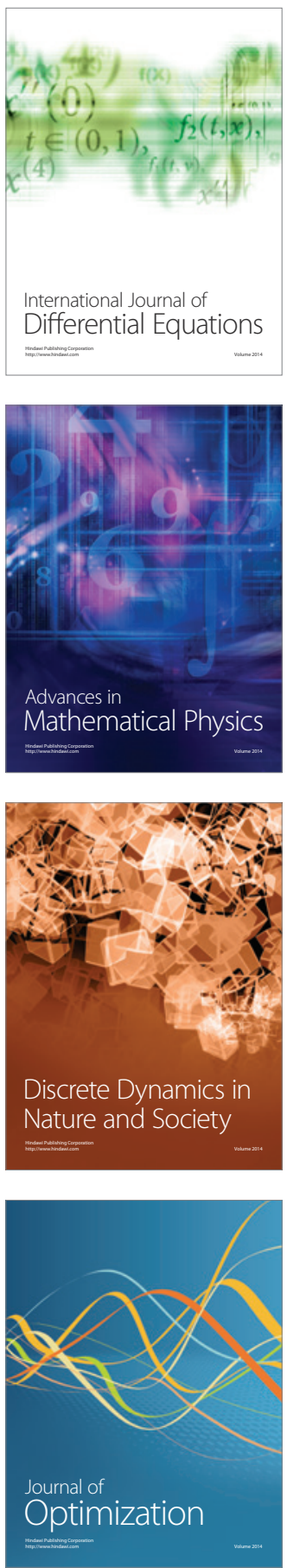